\title{
Una matemática diferente: la medición en dos grupos socioculturales de estudiantes universitarios. Estudio comparativo en la Universidad Nacional del Chaco Austral *
}

\author{
Tatiana Edith Vergara*
}

\section{Resumen}

Este artículo presenta un análisis sobre la medición como una actividad matemática básica y universal (Bishop, 1999). El desarrollo de este análisis se realizó a través de la indagación en dos grupos focales de estudiantes universitarios: el primero, de origen europeo (latinos y eslavos), y, el segundo, provenientes de Sudamérica (guaraníes). El objetivo de este estudio es establecer si el procedimiento de la medición adquiere rasgos particulares en función de las diferencias culturales de los sujetos que la realizan. La comparación permite observar las dificultades en el uso de las magnitudes continuas y la influencia del contexto en las respuestas de uno de los grupos.

\footnotetext{
* Artículo recibido el 27 de junio de 2014. Aceptado el 12 de septiembre de 2014.

* Tatiana Edith Vergara es Profesora en Física. Becaria de investigación en el proyecto "La incidencia de las ideas matemáticas y las nociones sobre la realidad natural del contexto sociocultural en la educación universitaria". Docente de la cátedra Química General, UNCAUS, Argentina. Maestranda de la Maestría en Educación en Cs. Experimentales y Tecnología, Facultad de Cs. Exactas, Físicas y Naturales, UNC.

Correo electrónico: tatianavergara@uncaus.edu.ar
} 


\section{Palabras clave}

Medición - Grupos socio-culturales - Estudiantes universitarios.

\section{Abstract}

This article presents an analysis about the measurement as a basic and universal mathematic activity (Bishop, 1999). Its development acquire different traits which depends on cultural and ethnic-social ownership of the individuals. We explored the measurement in two cultural groups, one of them from Europe native's college students (latins-slovenians) and other, natives from South America college students (guaraníes). The purpose is to establish whether the measurement has special features depending on the cultural differences of the subjects who performed. Our comparative analysis shows the difficulties to use continuous magnitudes and the influence of the context in the answers of one group.

\section{Keywords}

Measurement - Socio cultural group - College students.

\section{Introducción}

El presente trabajo forma parte del proyecto de investigación "La incidencia de las ideas matemáticas y las nociones sobre la realidad natural del contexto sociocultural en la educación universitaria" que se desarrolla en la Universidad Nacional del Chaco Austral (UNCAUS) bajo la dirección de la Dra. Ana Rosa Pratesi. Este proyecto tiene como finalidad indagar acerca de las causas que condicionan el aprendizaje de los alumnos centrando el interés en las nociones sobre conceptos científicos y las ideas sobre la realidad natural -teorías implícitas- que las personas poseen independientemente de su formación escolar y que inciden favorable o desfavorablemente en el aprendizaje de las ciencias y en el desempeño académico de los alumnos.

La importancia del estudio de estas teorías implícitas radica en su potencial utilización al momento de planificar un adecuado proceso de enseñanza que considere los saberes previos para desarticular las ideas erróneas y promover y enriquecer las ideas acertadas. La educación sistemática debe conocer estas ideas, ya sea para 
desmontarlas, ${ }^{1}$ o para validarlas y enriquecerlas. La construcción de un andamiaje significativo de desarrollo de nuevos aprendizajes debe establecerse dialécticamente con los saberes y las prácticas discursivas que los sujetos y sus comunidades han construido previamente.

Comprender la relación que existe entre el significado y las creencias que en la vida cotidiana los alumnos y sus grupos sociales atribuyen a los conceptos matemáticos y científicos, y la forma en que esos mismos conceptos luego interfieren en el aprendizaje de las disciplinas científicas -favoreciendo u obstaculizando el tránsito entre culturas-, permite conocer si se produce una hibridación léxica ${ }^{2}$. Esto significa, la convergencia de culturas y creencias diferentes que como fronteras porosas dejan su huella en el lenguaje o registran el tránsito de una cultura a otra. Más precisamente, entendemos la hibridación como:

Procesos socioculturales en las estructuras o prácticas discretas, que existían en forma separada, se combinan para generar nuevas estructuras, objetos y prácticas. [...] Estos procesos incesantes, variados, de hibridación llevan a relativizar la noción de identidad. El énfasis en la hibridación no sólo clausura la pretensión de establecer identidades "puras" o "auténticas". (García Canclini, 2003, pp. 2-3)

Explicar de qué manera estos significados inciden en el desempeño académico de los estudiantes a través de sus marcas discursivas, es una tarea importantísima en la comprensión de cómo operan las ideas previas, las creencias, en definitiva, la cultura de pertenencia de los sujetos en la adquisición de conocimientos científicos. Esta hibridación léxica también da cuenta del proceso de hibridación cultural que experimentaron los grupos socioculturales a los cuales pertenecen los estudiantes.

En otras palabras, la diversidad cultural supone lenguajes particulares de cada grupo y, por lo tanto, formas de razonamiento y representación del mundo diferentes. Es inevitable que estas diferencias culturales incidan en las modalidades de aprehensión de las nociones matemáticas y científicas propias del mundo académico, de allí la importancia de su estudio.

Para este trabajo se han conformado grupos integrados por estudiantes provenientes de medios culturales bajo diversas influencias, y se diseñó un instrumento que permitiera visibilizar las formas en que los sujetos desarrollan las siguientes actividades: asociación de objetos con números -contar-, simbolización del

\footnotetext{
${ }^{1}$ La acción de 'des-montar' implica des-membrar, des-entrañar la secuencia y el sentido de un saber, para comprenderlo y para des-cubrir a partir de su lógica por qué facilita o dificulta el aprendizaje de un concepto científico. Su significado, así, no adquiere una connotación negativa, sino de construcción a partir de la diversidad. Seguimos en este punto a Mortimer (2001) quien al definir los perfiles conceptuales se refiere a las diversas formas de ver y representar el mundo cuya comprensión está íntimamente relacionada con el contexto y las culturas.

2 Este concepto fue acuñado por la investigadora Silvia Noemí Sánchez en el marco de la formulación del PI № 30 UNCAUS 2012-2016. El enunciado da cuenta de que los procesos de hibridación cultural son múltiples y sus huellas pueden percibirse en el lenguaje, más específicamente en el léxico y los significados ambiguos, convergentes o combinados de muchos vocablos. La expresión "hibridación léxica" podría calificarse -según palabras de la misma investigadora- como un préstamo lingüístico proveniente de los Estudios Culturales. En el original usó comillas para dar cuenta de este último detalle.
} 
entorno espacial -localizar-, cuantificación de cualidades -medir-, creación de una estructura particular -diseñar-, establecimiento de reglas y procedimientos sociales jugar-, y los diversos aspectos cognitivos de investigar y conceptualizar el entorno y compartir esas conceptualizaciones -explicar- (Bishop, 1999). En este artículo abordaremos el análisis comparativo de la actividad 'medir' en dos grupos focales.

\section{Alan Bishop y la 'medición' como actividad matemática universal}

La tesis que sustenta este estudio considera a las Matemáticas ${ }^{3}$ como un producto cultural, idea propuesta por Alan Bishop en su libro Enculturación Matemática (1999). El propósito de este investigador es crear una nueva concepción de las Matemáticas que reconozca y demuestre su relación con la cultura, es decir demostrar la génesis cultural de las ideas matemáticas.

Partiendo de la educación matemática como una manera de conocer, Alan Bishop observa el conocimiento matemático desde una perspectiva cultural. Es así que la llama cultura matemática como forma breve de decir perspectiva cultural de las matemáticas o las matemáticas como fenómeno cultural.

Ahora bien, ¿qué entiende por cultura Bishop? Para responder esta pregunta, el autor realiza una revisión bibliográfica sobre distintos investigadores que han intentado definir el concepto de cultura. Para lo fines propuestos, Bishop retoma la definición de Stenhouse, quien sugiere que "la cultura consiste en un complejo de comprensiones compartidas mediante el cual los individuos pueden comunicarse entre sí" (1999, p. 22). Esto quiere decir que mientras las personas compartan ese complejo de comprensiones, pertenecerán al mismo grupo cultural, y en la medida en que ese complejo de comprensiones sea compartido por ese grupo de personas, esas comprensiones se convertirán en su cultura (Bishop, 1999). Por una parte, recurre a los trabajos de Morris Kline, quien desarrolla las matemáticas como un conjunto de ideas o complejo de comprensiones- que han influido en la formación de la sociedad moderna.

Por otra parte, Bishop retoma algunas ideas de Wilder, que:

Deja muy clara su postura sobre «las personas» cuando dice que "las personas que hacen matemáticas -los «matemáticos»- no sólo son los poseedores del elemento cultural conocido como matemáticas sino que, cuando se toman como un grupo por derecho propio, se pueden considerar los portadores de una cultura, en este caso la matemática" (p. 26). Por lo tanto, para Wilder, la cultura de ese complejo de comprensiones es la matemática y el grupo cultural es el de los «matemáticos». El interés de Wilder se centra en cómo evolucionan las ideas matemáticas y en quiénes «las hacen evolucionar». (p. 23)

\footnotetext{
${ }^{3}$ Bishop emplea la expresión de Matemáticas con mayúscula sugiriendo la creación de una nueva concepción de las Matemáticas que reconozca y demuestre su relación con la cultura.
} 
Posteriormente, Bishop sostiene que ninguno de los dos autores indica cuál es el papel de aquellas personas que no siendo matemáticos, se refieren a ellas "como parte de la civilización occidental (Kline) o de la cultura anfitriona (Wilder)" (p. 23). Su interrogante es saber cuál es el rol que desempeñan las personas que no pertenecen al grupo cultural de los matemáticos en la cultura matemática y cuál es su implicancia educativa. Esto lo lleva a ahondar en cuestiones referidas a los problemas de la educación matemática y en encontrar maneras educativamente significativas de relacionar a las personas y su cultura matemática.

A partir de un libro publicado por Gay \& Cole (1967), en el cual se desarrolla una investigación sobre las dificultades de los niños Kpelle para abordar conceptos y procesos propios de las nuevas matemáticas en las escuelas occidentalizadas, Bishop manifiesta su interés en trabajar con estos estudios culturales a fin de rescatar las similitudes en cuanto a ideas y actividades matemáticas entre grupos de diferentes culturas.

Con este objetivo, en un primer momento, Bishop consideró cuatro áreas principales de las matemáticas: número, medida, geometría y lenguaje/lógica, en donde se podrían encontrar similitudes a través de un análisis transcultural. Críticas posteriores confirmaron que estas áreas no eran suficientes para ahondar en los aspectos sobre los que se proponía indagar, como tampoco resultaba adecuado el abordaje de las similitudes a partir de temas particulares. Por esta razón, este autor decide implementar seis actividades matemáticas universales que ayuden a definir tanto las ideas matemáticas como los procesos que estas implican, bajo la hipótesis "de que el carácter de estos productos es muy posible que difiera de una cultura a otra" (Bishop, 1999, p. 42).

En primer lugar, definió aquellas actividades que si bien están relacionadas con el número, lo hacen desde perspectivas diferentes: contar y medir. En segundo término, aquellas que se refieren a la estructuración espacial y que han permitido un abundante desarrollo de ideas matemáticas, en particular, las ideas geométricas: localizar y diseñar. Por último, aquellas actividades que implican el vínculo con nuestro entorno social, ya que la cultura no se limita solo a la relación con el entorno físico: jugar y explicar.

En cuanto a la actividad universal matemática de medir, Bishop la define como aquella que se ocupa de comparar, ordenar y cuantificar cualidades que tienen valor e importancia. Dado que no todas las culturas valoran las mismas cosas en la misma medida, el entorno local inmediato es el que proporciona las cualidades que se han de medir, además de las unidades de medida.

Explica también que antes de que se desarrollen unidades de medición, existe una necesidad cultural evidente de que el lenguaje sea capaz de expresar cualidades mediante algún método comparativo y ordenado. La medición está relacionada con ideas como «más que» $y$ «menos que», porque la necesidad de medir solo se plantea si se quieren comparar dos o más fenómenos. Al compararse más de dos o tres objetos 
se desarrolla otra idea, la de ordenación. La estimación 'a ojo' es una técnica no verbal que se emplea para poner objetos en orden, pero si una cualidad crece en importancia y aumenta el número de objetos, el lenguaje desarrolla tanto palabras para los números ordinales (primero, segundo, etc.), como la «objetivación» de la cualidad (de pesado a más pesado, a peso). En conclusión, la actividad matemática de medir desempeña el papel de comparar cosas a partir de una cualidad compartida, partiendo de comparaciones muy simples de a pares, hasta de 'a muchos', y de unidades prácticas a las normalizadas, hasta llegar a un sistema complejo de unidades. Y a diferencia de la actividad matemática de contar, cuyo aspecto principal es su carácter discreto, la medición comprende la concepción de la cualidad como una cantidad continua.

A la vez, Bishop (1999) puntualiza en los conceptos organizadores que permitirían elaborar el currículum de matemáticas a partir de las seis actividades matemáticas propuestas. En este caso, presentamos los conceptos involucrados para el desarrollo de la actividad de medición (figura 1 ).

Figura 1: Conceptos organizadores del currículum para el desarrollo de la actividad matemática 'medir' (Bishop, 1999)

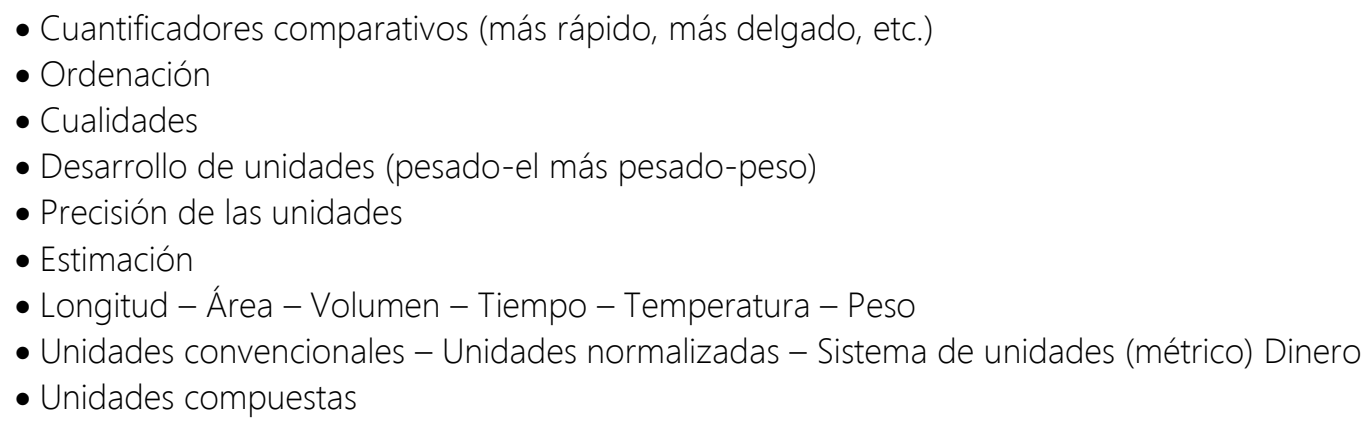

\section{La medición en otros referentes}

Nos parece interesante destacar las nociones de Castillo Mateo (2012), quien caracteriza la medición como el proceso por el cual se establece una medida. A su vez recurre a la definición de Callís ( Callís, 2002, citado en Castillo Mateos, 2012) para definirla como "la aplicación tecnológica y procedimental que tiene por objeto la obtención de la medida, o sea, es el conjunto de operaciones físicas o mentales a realizar, con o sin instrumentos, con el fin de determinar la medida de un objeto" (p.25). Además, Castillo Mateo sostiene que la medición de una determinada magnitud puede efectuarse por diversos métodos, como la utilización de algún instrumento de medición, comparación directa del objeto a medir con la unidad, comparación del objeto con otro del cual conocemos su medida -referente-, o bien mediante alguna técnica indirecta. Para concluir, este autor afirma que la medición resulta un proceso de 
comparación y ordenación a fin de establecer el significado del valor numérico obtenido en relación al contexto y a otras medidas efectuadas.

Rey Muñoz \& Aroca Araujo (2011) afirman que el desarrollo del sentido de la medida implica la participación de aspectos geométricos, semejanza en mediciones indirectas y los aspectos aritméticos. A su vez, involucra la estimación, entendida por Bright (1976), citado en Rey Muñoz \& Aroca Araujo (2011), como el proceso de llegar a una medida sin la ayuda de instrumentos, lo que convierte a la estimación en un proceso de suma importancia, ya que permite acceder a complejas técnicas de medición -Posada (2007) citado en el mismo artículo anterior-. Segovia Alex \& Castro Martínez (2009) también nos acercan una definición de estimación como "juicio sobre el valor del resultado de una operación numérica o de la medida de una cantidad, en función de circunstancias individuales del que lo emite". Debido a la multiplicidad de usos y campos de aplicación del concepto de estimación, estos autores distinguen dos tipos fundamentales de estimación: Por un lado, la estimación en cálculo, referida a las operaciones aritméticas y juicios que pueden establecerse sobre sus resultados. Por otra parte, la estimación en medida, que involucra los juicios que pueden establecerse sobre el valor de una determinada cantidad, o bien la valoración que puede hacerse sobre el resultado de una medida. Y dentro de este criterio se distingue el concepto de magnitud para referirnos a la conceptualización propia de la matemática clásica como todo aquello que se puede medir y expresar por medio de una cantidad. A su vez, discriminan dos grupos de magnitudes de tipo cuantitativas: escalar o continua y discreta. Castillo Mateo (2012) caracteriza la magnitud escalar o continua como aquella que presenta infinitos términos entre dos intervalos $[n, n+1]$, de manera que siempre puede garantizarse la división de una cantidad de magnitud en un número de partes iguales, y también, que la medida siempre puede aproximarse indefinidamente. En cuanto a la magnitud discreta, es aquella en la que no existen valores intermedios entre intervalos de medida, como ocurre con los números enteros.

\section{Metodología}

En el inicio del proyecto de investigación en el que se enmarca este trabajo, se implementó una encuesta dirigida a alumnos ingresantes a la Universidad Nacional del Chaco Austral en 2012 en la cual se indagó acerca de su origen sociocultural y, en particular, sobre los idiomas que se hablan en sus grupos familiares.

A partir de este relevamiento y tomando como referencia a Bishop (1999), se elaboró un instrumento de interacción para trabajar con grupos focales. Con el fin de evitar que las actividades planteadas en este instrumento remitiesen a un ejercicio escolarizado, en el cual los alumnos deberían exponer los conocimientos aprendidos en la educación formal, se formuló una serie de problemas directamente relacionados con su vida cotidiana. La aplicación de este instrumento se realizó en grupos focales, de tres a nueve integrantes, para que en la elaboración colectiva fuese posible captar las 
diferentes formas de abordar los problemas, aportar soluciones y argumentar por uno u otro razonamiento.

Para la conformación de los grupos focales se tomó como criterio el uso de lenguas de origen indoeuropeo por parte de los abuelos y padres de los alumnos, por un lado, y de lenguas amerindias, como el guaraní o el quechua, por otro. De esta forma, uno de los grupos estuvo integrado por estudiantes de ascendencia inmigratoria -europeos de origen latino o eslavo, grupo $\mathrm{M}-\mathrm{y}$ otro con descendientes de pobladores autóctonos americanos -descendientes de guaraníes, grupo G. En cuanto a la cantidad de integrantes, el grupo $\mathrm{M}$ se conformó con nueve participantes y el grupo $\mathrm{G}$ con cuatro participantes.

\section{La medición en los grupos culturales $\mathbf{G}$ y $\mathbf{M}$}

El instrumento diseñado nos permitió delimitar tres dimensiones o categorías de análisis que involucran la medición de tres magnitudes: la distancia, el tiempo y el consumo energético. Las actividades propuestas consistían en la descripción del recorrido que realizaban los estudiantes desde su casa hasta la Universidad. Las consignas que remitían a la actividad de medir eran las siguientes:

- ¿Qué distancia recorrieron en total? (teniendo en cuenta el recorrido de todos los participantes)

- ¿Cuánto tiempo ocuparon en hacer el recorrido en total? (teniendo en cuenta el tiempo ocupado por todos los participantes)

- ¿Qué cantidad de combustible se consumió en ese recorrido en total? (teniendo en cuenta el combustible consumido por todos los participantes)

Del análisis realizado a las discusiones de los grupos, se detectaron algunos de los conceptos organizadores propuestos en la figura 1 (p.6). Esto permitió definir las variables de comparación: desarrollo y utilización de unidades, estimación, cualidades y precisión.

\begin{tabular}{|c|c|c|c|c|}
\hline $\begin{array}{l}\text { Variables } \\
\text { de } \\
\text { comparación } \\
\text { Dimensiones }\end{array}$ & $\begin{array}{l}\text { Desarrollo y } \\
\text { utilización de } \\
\text { unidades }\end{array}$ & Estimación & Cualidades & Precisión \\
\hline Distancia & \multirow{3}{*}{$\begin{array}{l}\text { El Diccionario de } \\
\text { la Real Academia } \\
\text { Española la } \\
\text { define como } \\
\text { "cantidad que se } \\
\text { toma por medida } \\
\text { o término de }\end{array}$} & \multirow{3}{*}{$\begin{array}{l}\text { "Juicio sobre el } \\
\text { valor del } \\
\text { resultado de una } \\
\text { operación } \\
\text { numérica o de la } \\
\text { medida de una } \\
\text { cantidad en }\end{array}$} & \multirow{3}{*}{$\begin{array}{l}\text { La medición se } \\
\text { basa en la } \\
\text { comparación de } \\
\text { cosas a partir de } \\
\text { una cualidad } \\
\text { compartida. Se } \\
\text { entiende la }\end{array}$} & \multirow{3}{*}{$\begin{array}{l}\text { Para que la } \\
\text { experimentación } \\
\text { sea viable es } \\
\text { necesario el } \\
\text { empleo de } \\
\text { instrumentos de } \\
\text { medida a fin de }\end{array}$} \\
\hline Tiempo & & & & \\
\hline $\begin{array}{l}\text { Consumo } \\
\text { energético }\end{array}$ & & & & \\
\hline
\end{tabular}




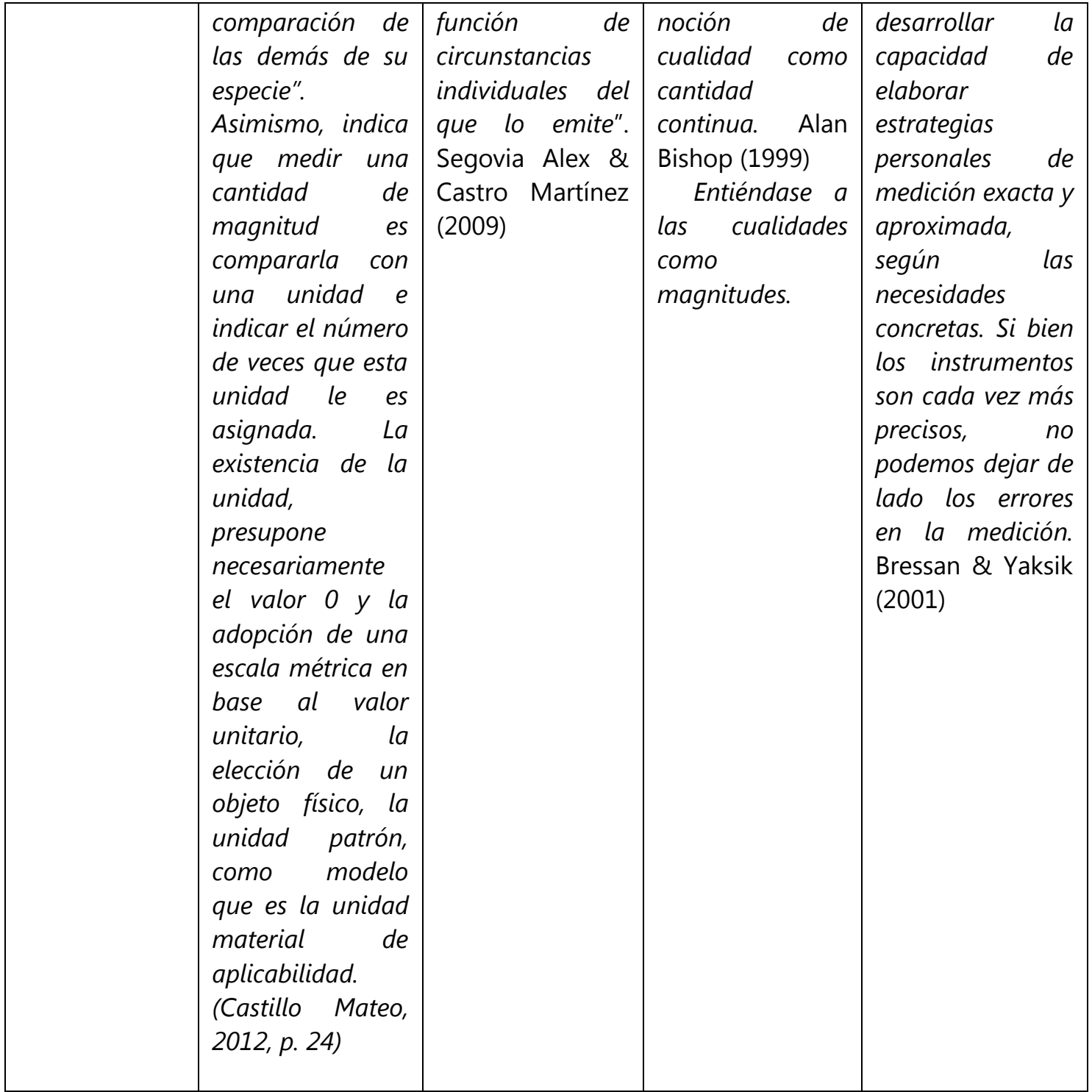

A partir de la delimitación de las dimensiones y las variables de comparación, se inició el análisis de los resultados de las discusiones.

\section{a. Medición de la distancia}

Frente a la pregunta propuesta por la moderadora: ¿Qué distancia recorrieron en total considerando el recorrido de todos juntos?, los dos grupos consideran las alternativas de obtener la distancia en metros -la distancia como magnitud continua- 0 en cuadras -al convertir la unidad de metros en cuadras, transforman a distancia en una magnitud discreta. En este caso, optan por las cuadras ya que, posiblemente, ante la dificultad, recurren a operaciones más simples. Esto significa que la estimación produce resultados aproximados dado que en su proceso, se opta por convertir o sustituir los datos por números sencillos, que sean fáciles de memorizar o bien accesibles para la realización de operaciones aritméticas mentales. Dada la prevalencia 
de la oralidad en el trabajo con los grupos focales -si bien los estudiantes podían escribir o calcular en papel, en la discusión predominó la oralidad-, con ello cobra relevancia la memoria, se hace necesario que los datos sean sencillos, o mantener cierto orden para facilitar su memorización. Por lo cual las magnitudes discretas resultan más accesibles en el cálculo mental de la distancia recorrida que las magnitudes continuas. Por consiguiente, esto transforma la actividad de medir en la actividad de contar: asignar un valor numérico a cada unidad de medida, en este caso, la cuadra. La conversión entre metros y cuadras que manejan ambos grupos es: 1 cuadra igual a 100 metros. Como se mencionó con anterioridad, Castillo Mateo (2012) afirma que los procesos de estimación de medidas involucran una serie de destrezas previas que facilitarían la realización de las estimaciones; una de ellas es el referente, es decir, conocer la medida de cantidades que resultan muy próximas. Se puede decir entonces que los participantes, al realizar el acuerdo de asignar a una cuadra la medida de $100 \mathrm{~m}$, hacen uso de referentes interiorizados como el conocimiento de que la longitud estándar de una cuadra es de 100 m, aunque sabemos que no siempre mantienen dicha medida. Veámoslo en los diálogos:

\section{Grupo G:}

M: -¿Qué distancia recorrieron en total considerando el recorrido todos juntos?

E1G:-La distancia total...

E2G: - ¿En metros?

E1G: -Entró Física..., en metros sobre segundo [risas].

M: -¿Qué distancia recorrieron en total todos juntos?

E2G: -¿En cuadras sería? Yo recorro...

\section{Grupo M:}

M: -Ahora, lo que le pedimos es que calculen, teniendo en cuenta el recorrido de cada uno, ¿qué distancia se recorrió en total?

E1M: - ¿En metros?

E2M: - ¿Cuadras?

E3M: -¿Cuántos metros tiene una cuadra?

Todos los Alumnos: -Cien.

E4M: -De la residencia ${ }^{4}$ hasta acá ponele dos cuadras.

$\cdots$

E4M: -Entre nosotros dos, seis cuadras. Vayan sumando de a dos.

E1M: -Vamos sumando de a cuatro, en orden.

E4M: -Cada uno pone su nombre.

\footnotetext{
${ }^{4}$ Residencia: complejo de departamentos gratuitos destinado al hospedaje de estudiantes que se encuentran cursando estudios de nivel superior (terciario y universitario).
} 
M: -¿Qué distancia recorrieron en total?

E5M: -Diez, doce, catorce... (Suman).

E4M: -Sin contar distancias de acá al final.

E1M: -(Dan el resultado pero hay interferencias que no permiten distinguir lo que dicen) (...)- $191 / 2$ cuadras

En el siguiente ejemplo, este grupo recurre a una estrategia para realizar un cálculo más sencillo y rápido:

\section{Grupo M:}

E4M: -Entre nosotros dos, seis cuadras. Vayan sumando de a dos.

E1M: - Vamos sumando de a cuatro, en orden.

E4M: -Cada uno pone su nombre.

En cuanto a la precisión de la medición de la distancia, si bien los estudiantes no recurrieron a la utilización de algún instrumento de medida, sí lo hicieron en una consigna anterior cuando debían realizar un croquis de su recorrido hasta la universidad. El grupo $\mathrm{G}$ realizó el dibujo a mano alzada mientras que los integrantes del grupo $\mathrm{M}$ utilizaron elementos de geometría -una regla- para hacer el dibujo.

\section{b. Medición del tiempo}

En relación al tiempo que les demandaba realizar el recorrido desde su casa hasta la universidad, el grupo $\mathrm{M}$ recurre al auxilio de un instrumento -el reloj- para denotar precisión en la medición realizada que a su vez es expresada en la unidad de minutos. Además, recurren a una conversión: una cuadra equivale a 'tantos' minutos. Por lo cual, frente a la identificación de esta magnitud continua utilizan un instrumento y simultáneamente, reflejan la necesidad de trabajar con magnitudes de cálculo más sencillas como las discretas. Todo lo contrario sucede con el grupo G, ya que estos recurren permanentemente a términos imprecisos como 'ponele' -es decir," utilizan una expresión que alude a la idea de 'convengamos que...'), pero emplean la misma unidad de medida: minutos. Entonces, en lo referido al tiempo, acuden a la aproximación. Este concepto no es sinónimo de estimación, pero sí se relaciona con ella en el hecho de que la aproximación consiste en encontrar un resultado que sea lo más preciso posible para un determinado fin. "La aproximación enfatiza la cercanía al valor exacto y es totalmente controlable; se aproxima tanto como se desee" (Segovia Alex \& Castro Martinez, 2009) Concretamente, en los diálogos:

\section{Grupo M:}

M: -Eh, bueno ¿cuánto tiempo ocuparon en hacer el recorrido en total? Lo mismo pero en vez de distancias, tiempo. 
E1M: - Y, pongamos las cuadras y tiempo.

(No se entiende muy bien lo que dicen porque hablan todos juntos. Luego suman veintiuno, veinticuatro, veintiséis y seis más, treinta y dos).

M: -¿Cómo hicieron para hacer ese cálculo, cuántos minutos? No miraron el reloj cuando salían.

E1M: -Yo miro el reloj.

M: -Vos mirás el reloj. ¿Cuando saliste y cuando entraste?

E1M: -Sí, yo siempre miro la hora y cuando más tardo es en cruzar la calle.

E4M: -Más cuando llegamos tarde.

\section{Grupo G:}

M: ¿¿Cuánto tiempo ocuparon para hacer el recorrido?

E1G:- $-Y$... cinco minutos como mucho, ponele tres minutos...

E2G: -De cinco a diez minutos.

[Risas]

E3G: -Un minuto y medio. Más rápido, si voy llegando tarde...

E3G: - ¿Y en total el tiempo que recorrimos...?

M: -Sí, el de todos también.

E1G: -Ponele diez.

E2G: -Ponele diez.

Otro aspecto relevante en el concepto del tiempo es la recurrencia a la noción de linealidad en ambos grupos. Cladellas (2009) nos explica que las distintas culturas conciben el tiempo de diversas maneras. En general, existen dos concepciones sobre el tiempo: la lineal y la circular. En las culturas occidentales originarias, el tiempo se consideraba cíclico, marcado por los ritmos de la siembra y de la cosecha, por los solsticios, por los movimientos del sol, y otros astros, y además se conmemoraban fiestas en relación a estos eventos naturales. Por el contrario, en la actualidad la noción de tiempo en la cultura occidental es lineal (pasado, presente y futuro). Lo frecuente en nuestra cultura es que el tiempo se mida con relojes y calendarios. A su vez, el uso del reloj ha generado la percepción del tiempo como regular y predecible. Tambien existen marcas fijas que dividen el tiempo en intervalos regulares: minutos, horas, meses, etc.

\section{c. Medición del consumo energético}

Ante la consigna "¿Cómo medirían la energía que consumieron a pie para llegar a la universidad?", los dos grupos recurren a la unidad caloría para expresar ese consumo energético (magnitud continua) pero surgen discrepancias en cuanto a la interpretación de la consigna por ambos grupos.

Uno de los participantes del grupo $G$ interpreta, en un primer momento, la medición del consumo de energía como sinónimo de cansancio -es decir, realiza la 
conversión de una magnitud de tipo cuantitativa a una de tipo cualitativa-, por lo cual, se podría pensar que para este participante una unidad de medida sería el 'cansancio'. Luego, sostiene que la energía consumida debería convertirla en calorías consumidas utilizando tablas de conversión- pero considerando diversos factores externos que inciden en la operación de medición, como la temperatura, la velocidad, el viento, etc. Estas variables inciden en la energía consumida imposibilitando su medida exacta:

E2G: - Y hay que tener en cuenta, que para calcular la energía consumida, por cada uno, varía de persona en persona, no es... La energía no es una ciencia exacta, no podés utilizar, podes calcular, cómo se llama, en condiciones ideales que todos tengan el mismo peso, la estatura también, pero si lo llevamos a la realidad todas las personas son distintas, no podés, tenés que ir evaluando la velocidad, el peso, la temperatura...

En cuanto al grupo $\mathrm{M}$, inmediatamente responden que medirían la energía consumida mediante la unidad de caloría y consultan a la moderadora si los cálculos deben ser exactos. Ofrecen su definición -aludiendo a saberes escolares- sin dar demasiadas explicaciones al respecto a diferencia del grupo anterior:

M: -Bueno, ehm...ustedes que vinieron a pie, ¿cómo medirían la energía que consumieron para hacer el trayecto, para recorrer esa distancia, tiempo, cómo medirían? Supongan una pregunta si el chico viene en colectivo o en moto, ¿cuánto combustible puede haber consumido en ese trayecto? y en el caso de los que vienen a pie ¿cómo medirían la energía? ¿Cuánta energía gastaron ustedes para hacer este trayecto?

E1M: - ¿En calorías? Ehm...

M: - ¿Cómo sería?

E5M: - ¿Tiene que ser exacto el cálculo?

M: -No, no, la pregunta es cómo medirían la energía que gastaron. ¿Qué quieren decir con calorías?

E4M: -O sea, no tenemos que hacer un cálculo de cuánta energía.

M: - ¿Cómo la medirían?

E4M: -Sí, está bien, calorías.

E6M: -Con calorías totales, digamos.

M: - Y, ¿qué es calorías?

E5M: -Caloría vendría a ser...

E1M: -Caloría es la cantidad de calor que se necesita para elevar un centímetro...

E4M: -... un grado...

E1M: -... un grado... ehm...

E4M: -Celsius, de un gramo de agua. 


$$
\begin{aligned}
& \text { E1M:-Ajá. } \\
& \text { M: -¿Cómo? }
\end{aligned}
$$

E1M: -O sea, la cantidad de calor que se necesita para elevar un gramo de agua a un grado Celsius, a un grado centígrado, sería.

M: -y en ustedes, eso ¿cómo se vería?

(Risas)

E5M: -El calor que nosotros generamos para gastar energía, con eso se podría medir.

En ambos grupos, se puede decir que al identificar que no se trata de una magnitud discreta, se encuentran con la dificultad de no poder realizar estimaciones aproximadas sobre el consumo energético que se les solicita. En consecuencia, recurren a distintas argumentaciones a fin de resaltar la imposibilidad que implica un cálculo mental sencillo y rápido para la estimación del consumo de energía. Apelan al cansancio, a la caloría, a la incidencia de múltiples factores -temperatura, viento, presión, etc.-, tablas de conversión, etc.

En este análisis también se observó que el grupo $G$ utilizó diminutivos que expresan la comparación de tamaño con otros puntos de referencias, por ejemplo:

E3G: -Sí, acá estaría la plaza...

E2G: -Esta es la dieciséis de la placita, después acá está la otra manzana.

E1G: - ¿No está tan chueca la calle esa?, ¿eh? (Risas)

E2G: -No, no te creas, ¿eh? (Risas).

E2G: -Acá ya hace la curvita esa.

E1G: -Sí, sí.

M: -A ver, ¿qué cantidad de calles cruzaste en el trayecto?

M: -Calculen todos.

E1G: -Yo cuatro calles.

M: -No, el que hizo el recorrido más largo.

E1G: $-A h$, él.

E2G: -Sí, cuatro calles, con todas estas calles son seis si tomás...

E1G: -Claro, tenés que contar que cruzás esta y esta...

E2G: -Claro, porque esta es una calle...

E1G: -Claro, tenés una, dos, tres...

E2G: -Si, y tomamos este trayectito como una más serían diez en total, pero si vos me decís en perpendicular son solo cuatro.

E1G: -Ajá, pasando el...

E2G:-En la manzana de la facultad. 
E1G: -Eh, sí, en un pedacito en la diecinueve, ${ }^{5}$ sí, diecinueve creo que es porque...

E1G: --Sigue la doce y acá ya está la ruta dieciséis, la dieciséis va así, ¿le vas a señalar ahí la ruta...?

Haciendo alusión a Aguirre et al. (2004), el uso del diminutivo posee dos valores claramente diferenciados: un valor semántico, con el que el diminutivo denota 'algo' de pequeñas dimensiones en relación a su valor habitual o en comparación con otro punto de referencia de mayores dimensiones -en el caso analizado, el punto de referencia tal vez sea la universidad-; y un valor prágmatico, por el que el diminutivo crea un clima de subjetividad al que acompañan toda un serie de elementos connotativos tales como la expresión de afectividad y ternura, la atenuación de aspectos negativos o la disminución de responsabilidad. Si tenemos en cuenta el concepto de estimación que venimos proponiendo desde el principio de este trabajo, los puntos o lugares de referencia constituyen nodos en relación a los cuales se aplican métodos o procesos de estimación para establecer posibles medidas de longitud (Rey Muñoz \& Aroca Araujo, 2011) o, como en este caso, reflejan -a través del lenguaje, y mediante la utilización de diminutivos- la comparación de tamaños en relación al punto de referencia que ellos utilizan.

Reynoso Noverón (2005) nos brinda un interesante estudio sobre el uso del diminutivo en español que nos permitirá puntualizar el uso de este recurso morfológico. El uso del diminutivo en español juega un papel importante en el fenómeno pragmático de la comunicación, dado que por este medio el hablante refleja tanto su idiosincracia cultural como sus intenciones comunicativas. El estudio realizado por este investigador, le permitió identificar tres grandes funciones pragmáticas que determinan tres tipos de relaciones que el sujeto establece con el mundo que lo rodea (figura 2):

Figura 2: Clasificación del uso del diminutivo según Reynoso Noverón (2005)

\section{ESQUEMA DE CLASIFICACIÓN SEMÁNTICO-PRAGMÁTICA}

\section{Valoración cuantificadora (Valoración de la dimensión de la entidad disminuida)}
a. Cuantificadora (uso referencial)
b. Descentralizadora
c. Centralizadora

II. Valoración cualificadora (Valoración de las cualidades de la entidad disminuida)
a. Negativa
b. Positiva

III. Valoración relacional (Valoración de las relaciones del hablante con las entidades en

\footnotetext{
${ }^{5}$ En la Ciudad de Presidencia Roque Sáenz Peña las calles se nombran con nombres propios (por ejemplo calle San Martín) y con números (la calle San Martín o calle 14).
} 


\section{el discurso)}
a. Irónica
b. Amortiguadora
c. Respetuosa

El objetivo de este estudio no es caracterizar cada una de estas valoraciones, por lo que se recomienta ver el trabajo del mencionado autor en su totalidad. Siguiendo dicha clasificación, se identificaron los usos del diminutivo en las interacciones del grupo focal G.

El uso por parte del participante E2G del término 'placita' focaliza el tamaño del objeto al que alude, otorgándole al diminutivo la función de cuantificador dimensional de tipo referencial (Reynoso Noverón, 2005).

En cuanto a los términos 'curvita', 'trayectito' y 'pedacito', cargan con una valoración también cuantificadora-centralizadora, es decir, enfoca el significado de la base a la que se aplica el diminutivo e intensifica el significado de esa base. El sujeto busca intensificar o aumentar tales características -'curvita', como una curva de pocas dimensiones; 'trayectito' como el recorrido de un trayecto corto, y 'pedacito' como la consideración de una porción pequeña de la calle a la cual se refiere (Reynoso Noverón, 2005).

En el caso del grupo $\mathrm{M}$, no se registró la utilización de diminutivos en el diálogo. Nuevamente, la presencia del contexto en los diálogos del grupo $G$ denota la importancia que este ítem tiene para ellos, en comparación con los integrantes del grupo M. En el caso del uso del diminutivo, continuamente los participantes del grupo $\mathrm{G}$ buscan caracterizar su entorno intensificando los detalles, a diferencia del grupo $\mathrm{M}$ que no emplea caracterizaciones tan particulares del medio.

Como resumen del análisis se presentan los siguientes cuadros:

\begin{tabular}{|c|c|c|c|c|}
\hline \multicolumn{5}{|c|}{ Medición de distancia } \\
\hline bles & Unidades & Estimación & Cualidades & Precisión \\
\hline Grupo G & $\begin{array}{c}\text { Conversión } \\
\text { de metros a } \\
\text { cuadras. }\end{array}$ & $\begin{array}{l}\text { Empleo de un } \\
\text { referente. }\end{array}$ & $\begin{array}{lr}\text { Necesidad de trabajar } \\
\text { con } & \text { magnitudes } \\
\text { discretas. Conversión } \\
\text { de la distancia: mag. } \\
\text { Continua } \quad \rightarrow \text { mag. } \\
\text { Discreta. }\end{array}$ & $\begin{array}{l}\text { Utilización } \\
\text { diminutivos. }\end{array}$ \\
\hline $\begin{array}{c}\text { Grupo } \\
\mathbf{M}\end{array}$ & $\begin{array}{c}\text { Conversión } \\
\text { de metros a } \\
\text { cuadras. }\end{array}$ & $\begin{array}{l}\text { Empleo de un } \\
\text { referente. }\end{array}$ & $\begin{array}{ll}\text { Necesidad de trabajar } \\
\text { con } & \text { magnitudes } \\
\text { discretas. Conversión } \\
\text { de la distancia: mag. } \\
\text { Continuar } \rightarrow \text { mag. } \\
\text { Discreta. }\end{array}$ & ------ \\
\hline
\end{tabular}




\begin{tabular}{|c|l|l|l|lr|}
\hline \multirow{2}{*}{$\begin{array}{c}\text { Vles } \\
\text { Grupo }\end{array}$} & Unidades & Estimación & \multicolumn{1}{|c|}{ Cualidades } & \multicolumn{2}{|c|}{ Precisión } \\
\cline { 2 - 6 } Grupo G & $\begin{array}{l}\text { Empleo de la } \\
\text { unidad } \\
\text { minutos. }\end{array}$ & $\begin{array}{l}\text { Recurren a la } \\
\text { experiencia } \\
\text { directa, al } \\
\text { entorno. }\end{array}$ & $\begin{array}{l}\text { Magnitudes continuas, } \\
\text { imposibilidad de } \\
\text { cálculos exactos. } \\
\text { Tiempo lineal. }\end{array}$ & $\begin{array}{l}\text { Utilización } \\
\text { expresiones } \\
\text { imprecisas como } \\
\text { 'más rápido', 'más } \\
\text { fuerte', etc.. }\end{array}$ \\
\hline $\begin{array}{c}\text { Grupo } \\
\text { M }\end{array}$ & $\begin{array}{l}\text { Empleo de la } \\
\text { unidad } \\
\text { minutos. }\end{array}$ & $\begin{array}{l}\text { Trabajo con } \\
\text { cálculos } \\
\text { sencillos }\end{array}$ & $\begin{array}{l}\text { Necesidad de emplear } \\
\text { magnitudes discretas. }\end{array}$ & $\begin{array}{l}\text { Utilización de un } \\
\text { instrumento de } \\
\text { medida (reloj) } \\
\text { sugiere la búsqueda } \\
\text { objetividad y } \\
\text { exactitud. }\end{array}$ \\
\hline
\end{tabular}

\begin{tabular}{|c|c|c|c|c|}
\hline \multirow{2}{*}{$\begin{array}{l}\text { Varia } \\
\text { bles } \\
\text { Grupo }\end{array}$} & \multicolumn{4}{|c|}{ Medición del consumo energético } \\
\hline & Unidades & Estimación & Cualidades & Precisión \\
\hline Grupo G & $\begin{array}{c}\text { Unidad } \\
\text { de medida } \\
\text { 'cansancio' } \\
\text { Conversi } \\
\text { ón a calorías } \\
\text { (por medio } \\
\text { de tablas) }\end{array}$ & $\begin{array}{l}\text { Intento de } \\
\text { definir una } \\
\text { fórmula. }\end{array}$ & $\begin{array}{c}\text { Conversión de } \\
\text { cualidades cuantitativas a } \\
\text { cualitativas mediante } \\
\text { cambio de unidades } \\
\text { (calorías } \rightarrow \text { cansancio) } \\
\text { Transformación de } \\
\text { magnitud cualitativa a } \\
\text { cuantitativa por medio de } \\
\text { tablas de conversión } \\
\text { (cansancio } \rightarrow \text { calorías) }\end{array}$ & $\begin{array}{l}\text { No es posible } \\
\text { realizar un cálculo } \\
\text { exacto por la } \\
\text { influencia de } \\
\text { factores externos. }\end{array}$ \\
\hline $\begin{array}{c}\text { Grupo } \\
\mathbf{M}\end{array}$ & $\begin{array}{c}\text { Empleo } \\
\text { de la unidad } \\
\text { 'caloría }\end{array}$ & ------- & ------ & $\begin{array}{c}\text { No aportan } \\
\text { valores, solo } \\
\text { definición de la } \\
\text { unidad de medida. }\end{array}$ \\
\hline
\end{tabular}

\section{Discusión y conclusiones}

A partir de los resultados, se detectaron algunas estrategias utilizadas por los participantes a la hora de medir. Para ello, retomamos las estrategias de mayor uso sugeridas por Bressan \& Yaksik (2001) resumidas en la figura 4. 
Figura 4: Estrategias más utilizadas para medir. Bressan \& Yaksik (2001)

\begin{tabular}{|l|}
\hline Comparación directa \\
\hline Implica conocer si $a>b$ ó a < b ó a $=\mathrm{b}$, comparándolas en base a la percepción \\
visual directa o bien por superposición de las cantidades a comparar. \\
\hline Comparación indirecta \\
\hline Implica utilizar instrumentos (al principio el mismo cuerpo o partes de él), o la \\
estimación. Se distinguen dos procedimientos: \\
1. El sujeto utiliza un elemento b como intermediario de manera que con él compara \\
la cantidad a medir como totalidad, sin partirla. \\
2. El sujeto utiliza unidades arbitrarias o convencionales pensando el objeto a medir \\
como descomponible en partes iguales. El proceso seguido al inicio en forma habitual \\
por los niños consiste en cubrir completamente con unidades la cantidad a medir en el \\
caso que sea posible (por ejemplo, en la medición de longitudes, áreas, capacidades y \\
volúmenes cubre todo con palillos, lentejas o bloques) para luego pasar a transportar esa \\
unidad, iterándola. En ambos casos obtiene un número que es la medida. \\
\hline Uso de fórmulas \\
En otras ocasiones las medidas de ciertas magnitudes físicas se obtienen también de \\
manera indirecta, por cálculo a través de fórmulas que implican el conocimiento de otras \\
medidas.
\end{tabular}

A partir de estos datos, se observó que el grupo $G$ recurre permanentemente a la estrategia de la comparación indirecta a partir de la estimación. Pero una estimación caracterizada por una fuerte experiencia sensorial directa del entorno inmediato. Vemos que tanto la influencia del contexto como el peso de la subjetividad expresada por algunos de los participantes en sus diálogos es una marcada diferencia entre el grupo $G$ y el grupo M. A la vez, en el grupo $G$, es recurrente el empleo de términos imprecisos -como la utilización de diminutivos, unidades desarrolladas por ellos mismos 'cansancio', etc.- que incrementan la carga subjetiva en sus diálogos.

En cuanto al grupo $\mathrm{M}$, en general, emplean la comparación indirecta por medio de la estimación y la utilización de instrumentos (reloj, regla). La estimación de este grupo se caracteriza por una influencia de conceptos del conocimiento científico que, si bien por momentos no es claro, provocan que sus respuestas tengan cierto nivel de objetividad y precisión. Esto se intensifica con el empleo de instrumentos de medición.

También es de destacar las dificultades que manifestaron ambos grupos al realizar mediciones que implicaban el trabajo con magnitudes continuas. Dado que la medición concibe la cualidad como una cantidad continua, permanentemente los participantes manifiestan la necesidad de transformar la cualidad continua en una cualidad discreta característica de la actividad de contar- para la realización de cálculos más sencillos y rápidos.

Se puede decir que a partir de la comparación de la actividad de los grupos, y aunque los grupos conformados tengan el mismo nivel educativo, al realizar el trabajo 
solicitado lo hicieron de maneras cualitativamente diferentes, por lo tanto, se puede inferir que esas diferencias provienen de aprendizajes diferentes de los adquiridos en su escolarización y que el ámbito universitario donde se desarrolló la experiencia no tuvo una influencia determinante.

\section{Agradecimientos}

A la Dra. Ana Rosa Pratesi y a la Lic. Silvia N. Sánchez y demás colegas del Proyecto de Investigación que integro, por su disponibilidad en la revisión y corrección del artículo asi como el incentivo y acompañamiento permanente en mis primeros pasos en la investigación.

\section{Abreviaturas o códigos empleados}

M: Moderador

E1G: Estudiante 1 grupo guaraní

E2G: Estudiante 2 grupo guaraní

E3G: Estudiante 3 grupo guaraní

E1M: Estudiante 1 grupo mixto

E2M: Estudiante 2 grupo mixto

E3M: Estudiante 3 grupo mixto

E4M: Estudiante 4 grupo mixto

E5M: Estudiante 5 grupo mixto

\section{Referencias}

AGUIRRE, C., ALBALÁ, M. J., \& MARRERO, V. (2004). "Mami, te quierito. La adquisición del diminutivo en español". IV Congreso Internacional sobre la Adquisición (págs. 120-144). Salamanca: Pulblicaciones de la Universidad de Salamanca de las Lenguas del Estado.

BISHOP, A. (1999). Enculturación matemática. La educación matemática desde una perspectiva cultural. Barcelona: Paidós.

BRESSAN, A. M., YAKSICH, F., (2001). "La enseñanza de la medida en la Educación General Básica. Obra colectiva de los docentes de la Red de escuelas de Campana", Bs. As.

CASTILLO MATEO, J. J. (2012). Estimación de cantidades continuas: Longitud y superficie. Tesis Doctoral. Granada.

CLADELLAS, R. (2009). El tiempo como factor cultural y su importancia socioeconómica: Estado del arte y líneas futuras. Intangible Capital, 5(2), 210-226.

GARCÍA CANCLINI, N. (2003). Noticias recientes sobre la hibridación. Revista transcultural de Música, (7). Disponible en http://www.sibetrans.com/trans/trans7/canclini.htm 
GAY, J. \& COLE, M. (1967). The New Mathematics in an Old Culture. Nueva York: Holt, Rinehart and Winston.

MORTIMER, E. F. (2001). Perfil conceptual: formas de pensar y hablar en las clases de ciencias. Infancia y aprendizaje, 4 (24), 475-490.

REY MUÑOZ, M. F., \& AROCA ARAUJO, A. (2011). Medición y estimación de los albañiles, un aporte a la educación matemática. 14 (1), 137-147.

REYNOSO NOVERÓN, J. (2005). Procesos de gramaticalización por subjetivización: El uso del diminutivo en el español. In Selected Proceedings of the 7th Hispanic Linguistics Symposium, ed. David Eddington, 79-86. Somerville, MA: Cascadilla Proceedings Project. Recuperado de www.lingref.com, document \#1088.

SEGOVIA ALEX, I., \& CASTRO MARTINEZ, E. (2009). La estimación en el cálculo y en la medida: fundamentación curricular e investigaciones desarrolladas en el Departamento de Didáctica de la Matemática de la Universidad de Granada. Electronic Journal of Research in Educational Psychology, 7(1), 499-536. 